\title{
Control of Natural Gas Catalytic Partial Oxidation for Hydrogen Generation in Fuel Cell Applications ${ }^{1}$
}

\author{
Jay T. Pukrushpan ${ }^{2}$, Anna G. Stefanopoulou ${ }^{2}$, Subbarao Varigonda ${ }^{3}$ \\ Lars M. Pedersen ${ }^{3}$, Shubhro Ghosh ${ }^{3}$, Huei Peng ${ }^{2}$
}

\begin{abstract}
A fuel processor that reforms natural gas to hydrogen-rich mixture to feed the anode field of fuel cell stack is considered. The first reactor that generates the majority of the hydrogen in the fuel processor is based on catalytic partial oxidation of the methane in the natural gas. We present a model-based control analysis and design for a fuel processing system that manages natural gas flow and humidified atmospheric air flow in order to regulate (i) the amount of hydrogen in the fuel cell anode and (ii) the temperature of the catalytic partial oxidation reactor during transient power demands from the fuel cell.
\end{abstract}

Linear feedback analysis and design is used to identify the limitation of a decentralized controller and the benefit of a multivariable controller. Further analysis unveils the critical controller cross-coupling term that contributes to the superior performance of the multivariable controller.

\section{Introduction}

Fuel Cells are considered for stationary (residential and commercial) and mobile (automotive and portable) power generation due to their high efficiency and environmental friendliness. Inadequate infrastructure for hydrogen refueling, distribution, and storage makes the fuel processor technology an important part of the fuel cell system for both stationary and mobile applications. For residential applications, fueling the fuel cell system using natural gas is often preferred because of its wide availability and extended distribution system [1]. Common methods of converting natural gas to hydrogen include steam reforming and partial oxidation. The most common method, steam reforming, which is endothermic, is well suited for steady-state operation and can deliver a relatively high concentration of hydrogen [2], but it suffers from a poor transient operation [3]. On the other hand, the partial oxidation offers several other advantages such as compactness, rapid-startup, and responsiveness to load changes [1], but delivers lower conversion efficiency.

A schematic of a typical partial oxidation-based FPS is shown in Figure 1. The majority of hydrogen is generated in a catalytic partial oxidation reactor (CPOX) where natural gas (mostly methane $\mathrm{CH}_{4}$ ) is combined with oxygen (in air) over

\footnotetext{
${ }^{1}$ Support is provided by NSF CMS-021332. Matching funds to these grants were provided by United Technologies

${ }^{2}$ J.T. Pukrushpan, A.G. Stefanopoulou and H. Peng are with the Department of Mechanical Engineering at the University of Michigan, Ann Arbor, Michigan.

${ }^{3}$ S. Varigonda, L.M. Pedersen and S. Ghosh are with the United Technologies Research Center, East Hartford, Connecticut.
}

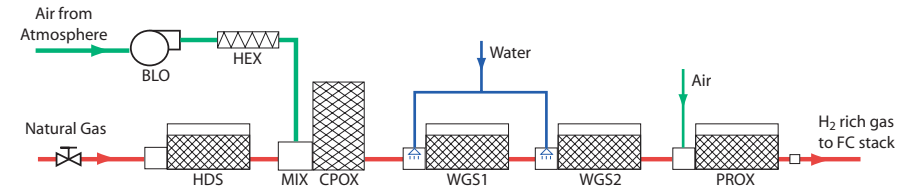

Figure 1: FPS components

a solid catalyst bed [4]. The two main factors that affect $\mathrm{CH}_{4}$ to $\mathrm{H}_{2}$ conversion efficiency of the CPOX are the catalyst bed temperature and the ratio of the reactants $\left(\mathrm{CH}_{4}\right.$ and $\left.\mathrm{O}_{2}\right)$ [5]. At proper CPOX operating temperature, the amount of hydrogen created depends on the supply rate of $\mathrm{CH}_{4}$ and the CPOX air to fuel ratio, more specifically, the oxygen to carbon ratio. This oxygen to carbon ratio also influences the amount of heat generated in the CPOX, which then affects the CPOX catalyst bed temperature. Carbon monoxide ( $\mathrm{CO})$, which poisons the fuel cell catalyst, is also created in the CPOX along with $\mathrm{H}_{2}$ and thus additional processing is needed to remove the CO. The typical CO removal process involves two reactors: water gas shift (WGS) and preferential oxidation (PROX) [6], which are represented, in this study, as if they perfectly remove the $\mathrm{CO}$ with the introduction of water and air. More details on the FPS chemical reactions are given in Section 2.

During changes in the stack current, the fuel processor needs to (i) quickly regulate the amount of hydrogen in the fuel cell stack (anode) to avoid starvation or wasted hydrogen [7] and (ii) maintain a desired temperature of the CPOX catalyst bed for high conversion efficiency [8]. Accurate control and coordination of the fuel processor reactant flows can prevent both large deviation of hydrogen concentration in the anode and large excursion of CPOX catalyst bed temperature. A control-oriented nonlinear model of the natural gas fuel processing system is developed in Section 3 with a focus on the dynamic behaviors associated with the flows and pressure in the FPS and also the temperature of the CPOX. The two main performance variables are the anode hydrogen mole fraction [9] and the CPOX catalyst bed temperature [5] and the two control actuators are the fuel $\left(\mathrm{CH}_{4}\right)$ valve command and the CPOX air blower command. The control problem is formulated in Section 4 and a linearized model derived in Section 5 is used in the control analysis and design.

Typical fuel processing systems rely on a decentralized (singleinput single-output) control of the air blower command to control CPOX temperature and of the fuel valve command to control the anode hydrogen concentration. In Section 6, an analysis using the relative gain array method confirms the appropriateness of the traditional input-output pairs for the decentralized control. The study also shows large interactions between the two loops at high frequencies and different op- 
erating conditions. These interactions can be more efficiently handled with multivariable control which is studied in Section 7. The linear quadratic optimal control method is used to design the controller (LQR) and the state estimator (LQG) that achieves a significant improvement in the CPOX temperature regulation as compared to the decentralized controller. It is shown in Section 8 that the regulation of the anode $\mathrm{H}_{2}$ mole fraction depends strongly on the speed of the fuel valve command while the improvement in the CPOX temperature regulation is due to the coordination of both inputs.

\section{Fuel Processing System (FPS)}

Figure 1 illustrates the components in a natural gas fuel processing system (FPS) [10]. Natural gas (mostly methane $\mathrm{CH}_{4}$ ) is supplied to the FPS from either a high-pressure tank or a high-pressure pipeline. The main air flow is supplied to the system by a blower (BLO) which draws air from the atmosphere. The air is then heated in the heat exchanger (HEX). The hydro-desulfurizer (HDS) is used to remove sulfur present in the natural gas stream $[1,11]$. The de-sulfurized natural gas stream is then mixed with the heated air flow in the mixer (MIX). The mixture is then passed through the catalyst bed inside the catalytic partial oxidizer (CPOX) where $\mathrm{CH}_{4}$ reacts with oxygen to produce $\mathrm{H}_{2}$. There are two main chemical reactions taking place in the CPOX: partial oxidation (POX) and total oxidation (TOX) $[5,12]$ :

$$
\begin{array}{ll}
(\mathrm{POX}) & \mathrm{CH}_{4}+\frac{1}{2} \mathrm{O}_{2} \rightarrow \mathrm{CO}+2 \mathrm{H}_{2} \\
(\mathrm{TOX}) & \mathrm{CH}_{4}+2 \mathrm{O}_{2} \rightarrow \mathrm{CO}_{2}+2 \mathrm{H}_{2} \mathrm{O}
\end{array}
$$

Heat is released from both reactions. However, TOX reaction releases more heat than POX reaction. The difference in the rates of the two reactions depends on the selectivity, $S$, defined as

$$
S=\frac{\text { rate of } \mathrm{CH}_{4} \text { reacting in } \mathrm{POX}}{\text { total rate of } \mathrm{CH}_{4} \text { reacting }}
$$

The selectivity depends strongly on the oxygen to carbon ratio $\left(\mathrm{O}_{2}\right.$ to $\left.\mathrm{CH}_{4}\right)$, denoted by $\lambda_{O 2 C}$, entering the CPOX [5]. Hydrogen is created only in POX reaction and, therefore, it is preferable to promote this reaction in the CPOX. However, the heat generated from POX reaction is not sufficient to maintain CPOX temperature. Thus, promoting TOX reaction is also required. Carbon monoxide $(\mathrm{CO})$ is also created along with $\mathrm{H}_{2}$ in the POX reaction as can be seen in (1). Since $\mathrm{CO}$ poisons the fuel cell catalyst, it is eliminated using both the water gas shift converter (WGS) and the preferential oxidizer (PROX). As illustrated in Figure 1, there are typically two WGS reactors operating at different temperatures $[3,6]$. In the WGS, water is injected into the gas flow in order to promote a water gas shift reaction:

$$
(W G S) \quad \mathrm{CO}+\mathrm{H}_{2} \mathrm{O} \rightarrow \mathrm{CO}_{2}+\mathrm{H}_{2}
$$

Note that even though the objective of WGS is to eliminate $\mathrm{CO}$, hydrogen is also created from the WGS reaction. The level of CO in the gas stream after WGS is normally still high for fuel cell operation and thus oxygen is injected (in the form of air) into the $\mathrm{PROX}$ reactor to react with the remaining $\mathrm{CO}$ :

$$
(\mathrm{PROX}) \quad 2 \mathrm{CO}+\mathrm{O}_{2} \rightarrow 2 \mathrm{CO}_{2}
$$

The amount of air injected into the PROX is typically twice the amount that is needed to maintain the stoichiometric reaction in $(5)[3,13]$.

\section{Control-Oriented FPS Model}

The FPS model is developed with a focus on the dynamic behaviors associated with the flows and pressures in the FPS and also the temperature of the CPOX.

Several assumptions are made in order to simplify the FPS model. Since the control of WGS and PROX reactants are not studied, the two components are lumped together as one volume and the combined volume is called WROX (WGS+PROX). It is also assumed that both components are perfectly controlled to obtain desired conversion and operating temperatures. Furthermore, because the amount of $\mathrm{H}_{2}$ created in WGS is proportional to the amount of CO that reacts in WGS (Reaction (4)), which in turn, is proportional to the amount of $\mathrm{H}_{2}$ generated in CPOX (Reaction (1)), it is assumed that the amount of $\mathrm{H}_{2}$ generated in the WGS is always a fixed percentage of the amount of $\mathrm{H}_{2}$ produced in the CPOX. The de-sulfurization process in the HDS is not modeled and thus the HDS is viewed as a storage volume. It is assumed that the pressures and compositions of the air entering the blower and of the natural gas entering the HDS are constant. Natural gas is considered as pure methane $\mathrm{CH}_{4}$. Additionally, any temperature other than the CPOX temperature is assumed constant and the effect of temperature changes on the pressure dynamics is assumed negligible. The volume of CPOX is relatively small and its dynamics are captured in the mixer. It is also assumed that all reactions are fast and reach equilibrium before the flow exit the reactors. Finally, all gases obey the ideal gas law and all gas mixtures are perfect mixtures. Figure 2 illustrates the simplified system and state variables used in the model.

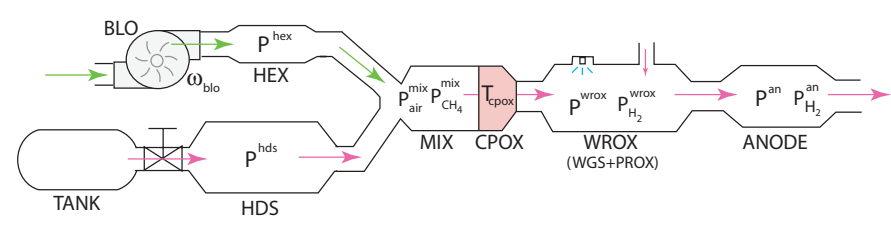

Figure 2: FPS dynamic model

The dynamic states in the model, shown also in Figure 2, are blower speed, $\omega_{b l o}$, heat exchanger pressure, $p^{\text {hex }}$, HDS pressure, $p^{h d s}$, mixer $\mathrm{CH}_{4}$ partial pressure, $p_{C H_{4}}^{m i x}$, mixer air partial pressure, $p_{\text {air }}^{\text {mix }}$, CPOX temperature, $T_{\text {cpox }}$, WROX (combined WGS and PROX) volume pressure, $p^{\text {wrox }}$, WROX hydrogen partial pressure, $p_{H_{2}}^{\text {wrox }}$, anode pressure, $p^{a n}$, and anode hydrogen partial pressure, $p_{\mathrm{H}_{2}}^{a n}$. We provide here a brief outline of the model. Although, we use the linearized model for the control analysis and design, the physical interpretation of the states helps in the control design and in interpreting the results.

The speed of the blower, $\omega_{b l o}$, is modeled as a first-order dynamic system with time constant $\tau_{b}$. The governing equation is

$$
\frac{d \omega_{b l o}}{d t}=\frac{1}{\tau_{b}}\left(\frac{u_{b l o}}{100} \omega_{0}-\omega_{b l o}\right)
$$

where $u_{b l o}$ is the blower command signal (range between 0 and $100)$ and $\omega_{0}$ is the nominal blower speed (3600rpm). The gas flow rate through the blower is modeled, $W_{\text {blo }}=f\left(\omega_{b l o}, \frac{p_{h e x}}{p_{a t m}}\right)$, using a blower map. Mass conservation with the ideal gas law 
through the isothermal assumption is used to model the pressure dynamics of the gas in all component volumes considered in the system. In any volume that does not involve any reaction, mixture composition is unchange and the total pressure of the gas is used as the state $\left(p_{h e x}\right.$ in HEX and $p_{h d s}$ in HDS). On the other hand, gas compositions in MIX, WROX and fuel cell anode (AN) changes due to reactions involved. The changes in gas composition in these volumes are described with additional partial pressures of the important species. In general, the pressure dynamics of a gas $n$ in a volume $m$ is governed by

$$
\frac{d p_{n}^{m}}{d t}=\frac{R T_{m}}{M_{n} V_{m}}\left(W_{n, \text { in }}^{m}-W_{n, \text { out }}^{m}\right)
$$

where $R$ is the universal gas constant, $V_{m}$ is the component gas volume, $M_{n}$ is the molar mass of species $n$, and $T_{m}$ is the temperature of the gas in the volume. The mass flow rate $W_{n, i n}^{m}$ is the rate (in $\left.(\mathrm{kg} / \mathrm{s})\right)$ of the species $n$ going into the volume $m$ which includes the species flow into the volume and the species produced (from the reaction) in the volume. The flow rate $W_{n, \text { out }}^{m}$ are the rate of species $n$ going out of the volume including the species flow rate exiting the volume and the rate of species reacted in the reaction.

The total flow rate, $W$, between two volumes is, in general, calculated from pressure differential, $p_{1}-p_{2}$, using the orifice equation with a turbulent flow assumption

$$
W=W_{0} \sqrt{\frac{p_{1}-p_{2}}{\Delta p_{0}}}
$$

where $W_{0}$ and $\Delta p_{0}$ are the nominal air flow rate and the nominal pressure drop of the orifice, respectively. The flow rate of a constituent species $n$ between the volumes is a function of the total gas flow and the mole fraction of the species $n$ in the upstream volume. The flow rate of fuel (natural gas) into HDS, $W_{h d s, i n}$, is, in addition, a function of the valve input, uvalve,

$$
W_{h d s, \text { in }}=\left(\frac{u_{\text {valve }}}{100}\right) W_{0, \text { valve }} \sqrt{\frac{p^{\text {tank }}-p^{h d s}}{\Delta p_{0, \text { valve }}}}
$$

where $p^{\operatorname{tank}}$ is the fuel tank or supply line pressure.

The conversion of the gases in CPOX is based on the reactions in (1) and (2) and the selectivity defined in (3), which is a function of the oxygen to carbon ratio in MIX, $\lambda_{O 2 C}$ :

$$
\lambda_{O 2 C}=y_{O_{2}}^{a t m} \frac{p_{a i r}^{m i x}}{p_{C H_{4}}^{m i x}}
$$

where $y_{\mathrm{O}_{2}}^{a t m}$ is the oxygen mole fraction of the atmospheric air. The energy conservation principle is used to model the changes in CPOX temperature.

$$
m^{c p x} C_{P}^{c p x} \frac{d T_{\text {cpox }}}{d t}=\left[\begin{array}{c}
\text { inlet } \\
\text { enthalpy } \\
\text { flow }
\end{array}\right]-\left[\begin{array}{c}
\text { outlet } \\
\text { enthalpy } \\
\text { flow }
\end{array}\right]+\left[\begin{array}{c}
\text { heat } \\
\text { from } \\
\text { reactions }
\end{array}\right]
$$

where $m^{c p x}(\mathrm{~kg})$ and $C_{P}^{c p x}(\mathrm{~J} / \mathrm{kg} \cdot \mathrm{K})$ are mass and specific heat capacity of the catalyst bed, respectively. The terms on the right hand side of (11) are determined based on the reactants and products gas of the CPOX reactions. Further details of the model are presented in [14].

\section{Control Problem Formulation}

As previously discussed, the main objectives of the FPS controller are (i) to protect the stack from damage due to $\mathrm{H}_{2}$ starvation (ii) to protect CPOX from overheating and (iii) to keep overall system efficiency high, which includes high stack $\mathrm{H}_{2}$ utilization and high FPS $\mathrm{CH}_{4}$-to- $\mathrm{H}_{2}$ conversion. Objectives (ii) and (iii) are related since maintaining the desired CPOX temperature during steady-state implies proper regulation of the oxygen-to-carbon ratio which corresponds to high FPS conversion efficiency.

Two performance variables that need to be regulated are the anode hydrogen mole fraction, $y_{H_{2}}$,

$$
y_{H_{2}}=\frac{p_{H_{2}}^{a n}}{p^{a n}}
$$

and the CPOX temperature, $T_{\text {срох }}$. They are chosen based on the following rationale. High $T_{\text {cpox }}$ can cause the catalyst bed to be overheated and be permanently damaged. Low $T_{\text {cpox }}$ results in a low $\mathrm{CH}_{4}$ reaction rate in the CPOX [5] and potential methane slip. Large deviations of $y_{H_{2}}$ are undesirable. On one hand, a low value of $y_{H_{2}}$ means anode $\mathrm{H}_{2}$ starvation $[9,16]$ which can permanently damage the fuel cell structure. On the other hand, a high value of $y_{H_{2}}$ means small hydrogen utilization which results in a waste of hydrogen.

In this control study, we assume that all $\mathrm{CH}_{4}$ that enters the CPOX reacts without any methane slip. Note that these assumptions reduce the validity of the model for large $T_{c p o x}$ deviations. The effect of the modeling error due to these assumptions can degrade the performance of the model-based controller. However, achieving one of the control goals, which is the regulation of $T_{\text {cpox }}$, will ensure that this modeling error remains small.

The actuator dynamics are ignored. The stack current, $I_{s t}$, is considered as an exogenous input that is measured. Since the exogenous input is measured, we consider a two degrees of freedom (2DOF) controller based on feedforward and feedback, as shown in Figure 3. The two control inputs, $u$, are the air

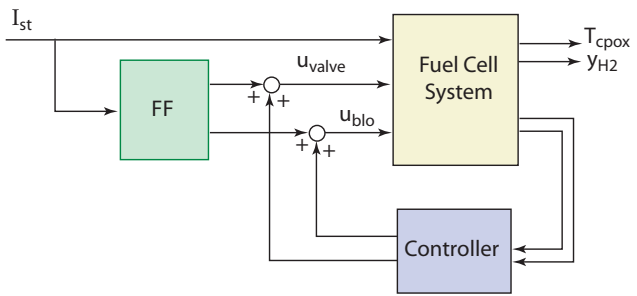

Figure 3: Feedback control study

blower signal, $u_{b l o}$, and the fuel valve signal, $u_{v a l v e}$. The feedforward terms that provide the valve and the blower signals that reject the steady-state effect of current to the outputs are integrated in the plant: $u^{*}\left(I_{s t}\right)=\left[\begin{array}{ll}u_{b l o}^{*}\left(I_{s t}\right) & u_{v a l v e}^{*}\left(I_{s t}\right)\end{array}\right]^{T}$. The value of $u^{*}$ is obtained by nonlinear simulation and can be implemented with a lookup table. The performance variable are the CPOX temperature, $T_{\text {cpox }}$, and the anode exit hydrogen mole fraction, $y_{\mathrm{H}_{2}}$. 


\section{Analysis of Linearized Models}

A linear model of the FPS is obtained by linearizing the nonlinear model. In this study, the desired steady-state is selected at stack $\mathrm{H}_{2}$ utilization $U_{H_{2}}=80 \%$ [13] and CPOX oxygento-carbon ratio $\lambda_{O 2 C}=0.6$. This condition results in the value of CPOX temperature, $T_{\text {cpox }}=972 \mathrm{~K}$ (corresponds to $\left.\lambda_{\mathrm{O} 2 \mathrm{C}}=0.6\right)$, and the value of anode hydrogen mole fraction, $y_{\mathrm{H}_{2}} \approx 9 \%$ (corresponds to $U_{\mathrm{H}_{2}}=80 \%$ ). The control objective is therefore to regulate $T_{\text {cpox }}$ at $972 \mathrm{~K}$ and $y_{\mathrm{H}_{2}}$ at 0.09 . This desired value of $T_{\text {cpox }}$ also agrees with the value published in the literature [15]. Static feedforward terms (illustrated in Figure 3) are included in the linear plant so that the steadystate $T_{c p o x}$ and $y_{H_{2}}$ are maintained at nominal value during changes in stack current. The linearization of the plant is denoted by

$$
\begin{aligned}
& \dot{x}=A x+B_{u} u+B_{w} w \\
& z=C_{z} x+D_{z u} u+D_{z w} w
\end{aligned}
$$

where the state, $x$, input, $u$, disturbance, $w$, and performance variables, $z$, are

$$
\begin{gathered}
x^{T}=\left[T_{\text {cpox }}, p_{H_{2}}^{\text {an }}, p^{a n}, p^{h e x}, \omega_{\text {blo }}, p^{h d s}, p_{C_{4}}^{\text {mix }}, p_{\text {air }}^{\text {mix }}, p_{H_{2}}^{\text {wrox }}, p^{\text {wrox }}\right] \\
w=I_{s t}, \quad u^{T}=\left[u_{\text {blo }} u_{\text {valve }}\right], \quad z^{T}=\left[T_{\text {cpox }} y_{H_{2}}\right]
\end{gathered}
$$

All variables in the linear plant above are perturbations from the nominal. The current input is in Ampere. The blower and the valve signals, $u_{b l o}$ and $u_{v a l v e}$, vary between 0 and 100. The outputs are the CPOX temperature in Kelvin and the anode hydrogen mole fraction in percent. In the transfer function form, we can represent the plant as

$$
z=\left[\begin{array}{ll}
G_{w} & G
\end{array}\right]\left[\begin{array}{l}
w \\
u
\end{array}\right]
$$

The nonlinear plant model is linearized at three different current (load) levels that correspond to the $30 \%, 50 \%$, and $80 \%$ of the plant power level. The Bode plots of the linear plants that are obtained from different system power levels are shown in Figures 4. For clarity, the units of current is (×10 Amp). Note that a right half plane (RHP) zero exists in the path

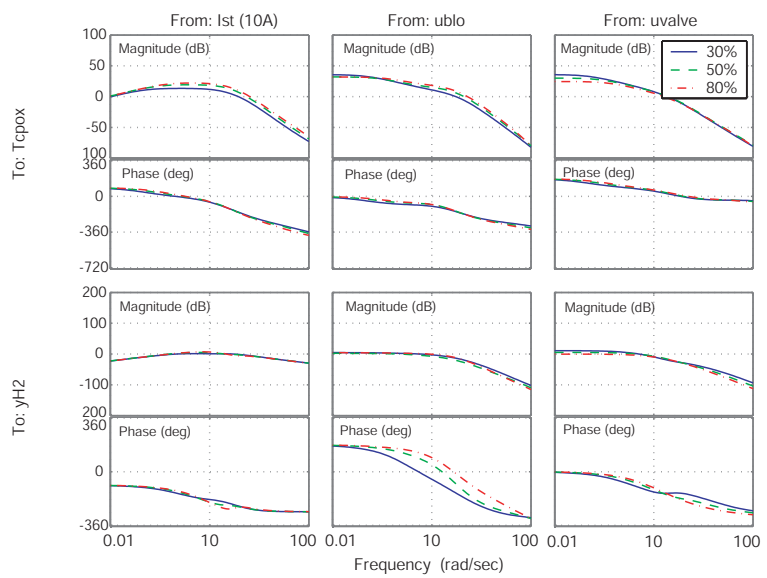

Figure 4: Bode plot of linearized models at 30\%, 50\%, and $80 \%$ power

$u_{\text {blo }} \rightarrow y_{H_{2}}$. Moreover, as can be seen in the frequency response of $u_{b l o} \rightarrow y_{H_{2}}$ in Figure 4, the RHP zero moves closer to the imaginary axis at low power level (30\%). The linearization of the system at the $50 \%$ power level is used in the control study in the following sections.

\section{Input-Output Pairing}

Two PI controllers can be used for the two-input two-output (TITO) plant. The resulting diagonal or decentralized controller structure is an effective control architecture for the TITO system unless there is a significant coupling between the two loops. Due to the non-minimum phase zero in the $u_{b l o} \rightarrow y_{H_{2}}$ transfer function, the preferred pairing choices are $u_{\text {blo }} \rightarrow T_{\text {cpox }}$ pair and $u_{\text {valve }} \rightarrow y_{\mathrm{H}_{2}}$ pair. This pairing choice is also confirmed by the relative gain array (RGA) matrix [17] of $G$,

$$
R G A=G \times\left(G^{-1}\right)^{T}
$$

at zero frequency:

$$
R G A(0 \mathrm{rad} / \mathrm{s})=\left[\begin{array}{cc}
2.302 & -1.302 \\
-1.302 & 2.302
\end{array}\right]
$$

The RGA can also be used to assess the loop interactions Large off-diagonal elements of the RGA matrix indicates large loop interactions. A plot of the magnitude difference between the diagonal and off-diagonal elements of the RGA matrices in Figure 5 shows that the interactions increase at high frequencies $[18,17]$. At low power levels, the values of the off-diagonal elements of the RGA matrix are even higher than the diagonal elements $\left(\left|R G A_{11}\right|-\left|R G A_{12}\right|<0\right)$, indicating large coupling. At these frequencies and power levels, we can expect poor performance from a decentralized controller.

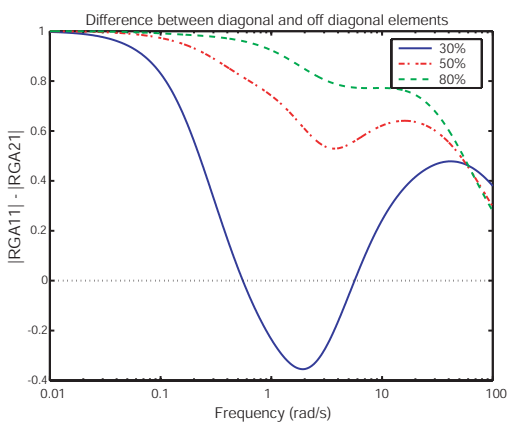

Figure 5: Difference between diagonal and off-diagonal elements of the RGA matrix at different frequencies for three power setpoints

After these observations, a PI controller in the fuel loop $\left(u_{\text {valve }} \rightarrow y_{\mathrm{H}_{2}}\right)$ is tuned to achieve fast response of $y_{\mathrm{H}_{2}}$ without saturating the valve. Then, to achieve fast regulation of $T_{\text {cpox }}$, we also tune the second PI for fast air loop while avoiding blower saturation. The performance of the decentralized controller with fast controller in both loops degrades significantly when the system operates at low power. The loop interaction dictates a bandwidth separation between the two loops. Since designing the air loop to be faster than the fuel loop is not feasible with a PI controller due to blower magnitude constraints, the air loop is detuned to be slower than the fuel loop. It can be seen that the speed of $T_{\text {cpox }}$ regulation has to be sacrificed to prevent the degradation effect of system interactions on the decentralized controller. 


\section{Multivariable Control}

In this section, we assess the improvement gained by a controller developed using a multivariable and model-based control design techniques. The controller is designed using linear quadratic (LQ) methodology.

To eliminate steady-state error, we first add to the controller the integrators on the two performance variables, $T_{\text {cpox }}$ and $y_{\mathrm{H}_{2}}$. The controller is designed with the objective of minimizing the cost function

$$
J=\int_{0}^{\infty} z^{T} Q_{z} z+q^{T} Q_{I} q+u^{T} R u d t
$$

where $Q_{z}, Q_{I}$, and $R$ are weighting matrices. The control law that minimizes (17) is in the form

$$
u=-K_{P}\left(\hat{x}-x_{d}\right)-K_{I} q=-K\left[\begin{array}{c}
\left(\hat{x}-x_{d}\right) \\
q
\end{array}\right]
$$

where $K$ is the control gain. Variable $x_{d}$ in (18) is a function of $I_{s t}$ and can be viewed as the desired value of the states that gives the desired value of $z=0$. In other words, the term $K_{p} x_{d}$ is an additional feedforward term that compensates for the changes in the output steady-state value due to the feedback. The value of $x_{d}$ can be found by simulation or by the linear plant matrices (13). In both cases, $x_{d}$ is not robustly accurate, nevertheless, the steady-state regulation is achieved by the integrators. The estimate of the plant state, $\hat{x}$, can be determined using an observer

$$
\begin{aligned}
& \dot{\hat{x}}=A \hat{x}+B_{u} u+B_{w} w+L(z-\hat{z}) \\
& \hat{z}=C_{z} \hat{x}+D_{z u} u+D_{z w} w
\end{aligned}
$$

where $L$ is the estimator gain determined based on LQG methodology.

The nonlinear simulation of the system with the decentralized PI feedback and with the output observer-based feedback is shown in Figure 6. The output feedback gives satisfactory performance in both $y_{H_{2}}$ and $T_{c p o x}$ regulations.
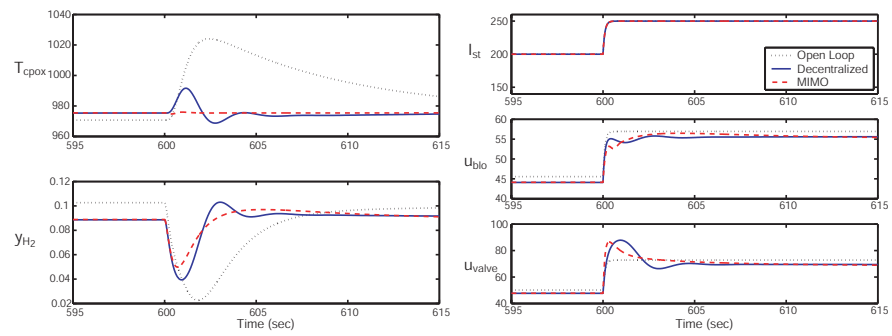

Figure 6: Comparison of decentralized PI controller and observer feedback in nonlinear simulation

\section{Insight Gained by the Multivariable Design}

The combination of the state feedback control (18) and the state observer (19) results in a model-based multivariable output-feedback controller. In transfer function form, the controller can be written as

$$
u=C_{w} w+C z=\left[\begin{array}{l}
C_{w 1} \\
C_{w 2}
\end{array}\right] w+\left[\begin{array}{ll}
C_{11} & C_{12} \\
C_{21} & C_{22}
\end{array}\right] z
$$

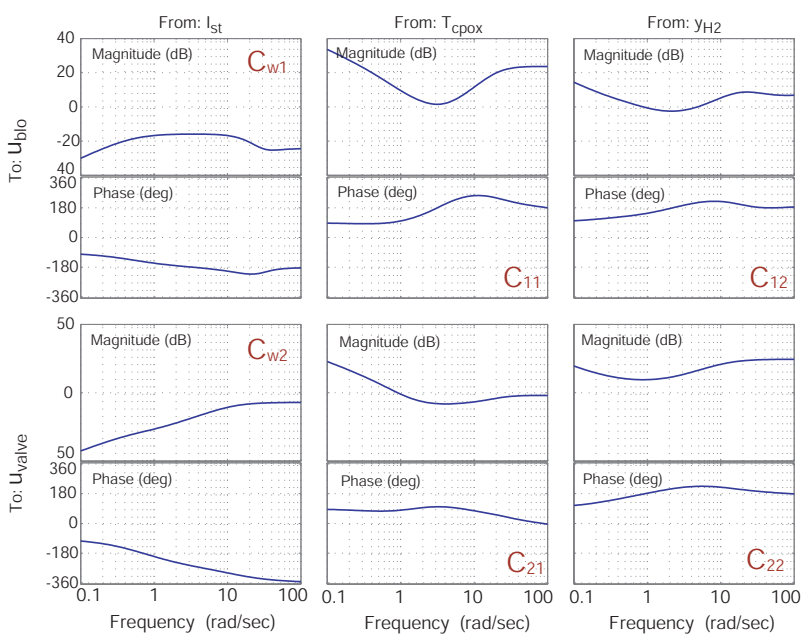

Figure 7: Frequency response of the controller

The Bode plot of each element of the controller is shown in Figure 7.

In an effort to simplify the feedback controller for gain scheduling and implementation purposes, we investigate which crosscoupling term of the feedback contributes to the improvement by the MIMO controller. By zeroing out the cross-coupling term and plotting the closed loop frequency and time responses in Figures 8 and 9, we can see that the performance of the full controller is maintained when $C_{21}=0$ (triangular MIMO controller). However, the performance degrades when $C_{12}=0$ (diagonal MIMO controller). Thus it is clear that the $C_{12}$ term is the critical cross-coupling term that provides the MIMO control improvement.

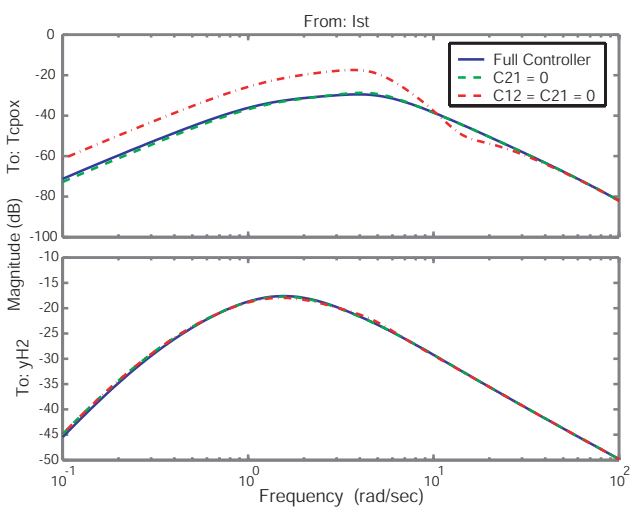

Figure 8: Closed-loop frequency response for analysis of elements in the feedback controllers
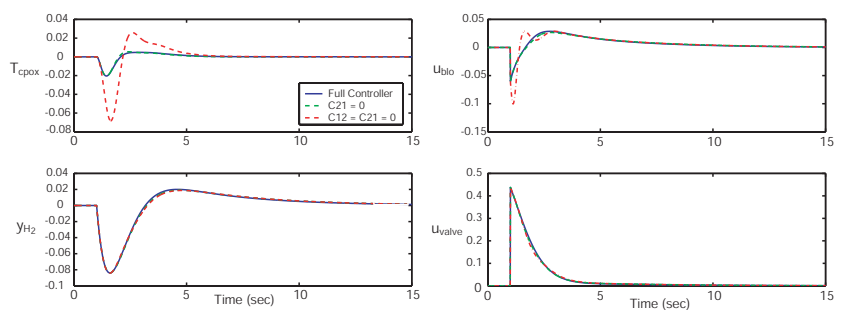

Figure 9: Closed-loop time response for analysis of elements in the feedback controllers 


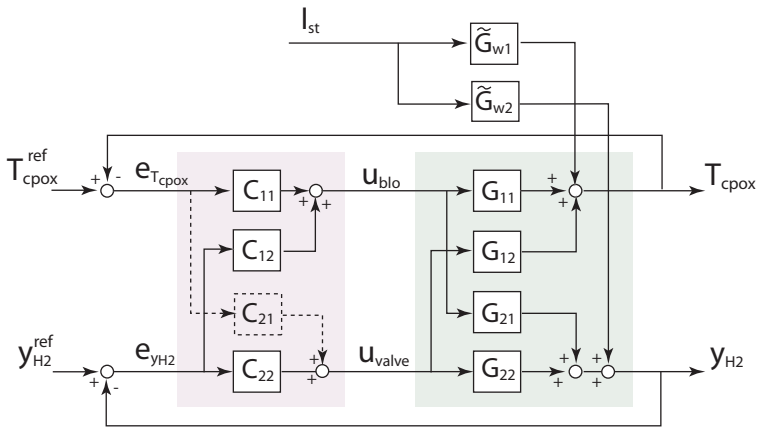

Figure 10: Block Diagram of FPS plant and simplified controller

The importance of $C_{12}$ is interpreted as follows. Following Figure 10, the current disturbance, $I_{s t}$, affects more $y_{H_{2}}$ than $T_{\text {cpox }}$ during fast transient as can be seen by the large highfrequency magnitude of the transfer function from $I_{s t}$ to $y_{H_{2}}$ (Figure 11) for the plant with feedforward control: $\tilde{G}_{w}=$ $G_{w}+G C_{w}$. The valve signal, $u_{v a l v e}$, tries to reject the effect

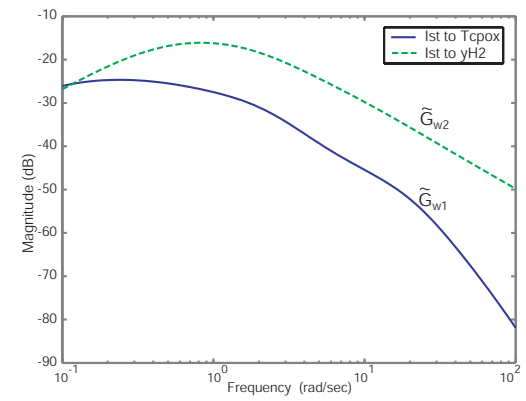

Figure 11: Frequency magnitude plot of the plant with dynamic feedforward part of the controller $\tilde{G}_{z w}$ in (8)

of $\tilde{G}_{w 2} I_{s t}$ to $y_{H_{2}}$ (see Figure 10) by using the feedback $C_{22}$ term through $G_{22}$. The blower signal, on the other hand, cannot help reject the $\tilde{G}_{w 2} I_{s t}$ to $y_{H_{2}}$ through $G_{21} C_{12}$ because of the non-minimum phase zero of $G_{21}\left(z_{N M P}=3.07\right)$.

$$
\frac{\tilde{G}_{w 2} I_{s t}}{1+G_{21} C_{12}+G_{22} C_{22}} \simeq \frac{\tilde{G}_{w 2} I_{s t}}{1+G_{22} C_{22}}
$$

Indeed, Figure 7 shows that the magnitude of $C_{12}$ is low at frequencies close to that of the NMP zero. Meanwhile, the valve that tries hard to reject the $\tilde{G}_{w 2} I_{s t}$ to $y_{H_{2}}$ causes disturbances to $T_{\text {срох }}$ through the plant $G_{12}$ interaction. The controller cross-coupling term $C_{12}$ is thus needed to compensate for the effect of $u_{\text {valve }}$ to $T_{\text {cpox }}$ by partially cancelling $G_{12} C_{22}$ by $G_{11} C_{12}$ at certain frequencies.

$$
T_{\text {срох }}=\frac{G_{12} C_{22}+G_{11} C_{12}}{1+G_{11} C_{11}} e_{y_{H_{2}}} \approx 0 \Rightarrow C_{12} \approx-G_{11}^{-1} G_{12} C_{22}
$$

Note that this partial cancellation involves the plant elements $G_{11}$ and $G_{12}$ that do not change significantly for different power levels, as compared to $G_{21}$ (see Figure 4). Thus the benefit of the controller cross-coupling term $C_{12}$ is maintained in full range of operating power. If the air loop has high bandwidth, the $G_{11} C_{11}$ term can reject the disturbance by itself and, then, controller $C_{12}$ is not needed to cancel the interaction from the valve to $T_{\text {cpox }}$.
Figure 7 also verifies that $C_{21}$ does not contribute to the overall MIMO controller. The magnitude of $C_{21}$ is, in fact, relatively smaller than other feedback terms. At high frequencies where the effect of $\tilde{G}_{w 2} I_{s t}$ to $y_{H_{2}}$ is large, the term $C_{21}$ is not used to help regulating $y_{H_{2}}$ because the deviation in $y_{H_{2}}$ is not reflected in $T_{\text {cpox }}$ measurement $\left(\tilde{G}_{w 1} I_{s t}\right.$ is small). At low frequencies where $I_{s t}$ affects $T_{\text {cpox }}, C_{21}$ may be used to help reduce $T_{\text {срох }}$ error but will cause disturbance to the well-behaved fuel loop, thus $C_{21}$ is also insignificant at low frequencies.

By comparing the response of decentralized PI controller in Figure 6 and that of the diagonal MIMO controller in Figure 9, we can see that the diagonal controller derived from the MIMO controller outperforms the decentralized PI controller. This is achieved as shown in Figure 12 because of the higher closed loop bandwidth of the air loop when compared with the one of the PI-based controller. As seen in Figure 7, the high-order $C_{11}$ term can have high bandwidth without having high gain and thus avoids blower saturation. This can not be achieved using a PI controller. Indeed, Figure 7 verifies that the gain of $C_{11}$ is low at the frequencies where loop-interaction is large (see Figure 5 for the loop interactions).

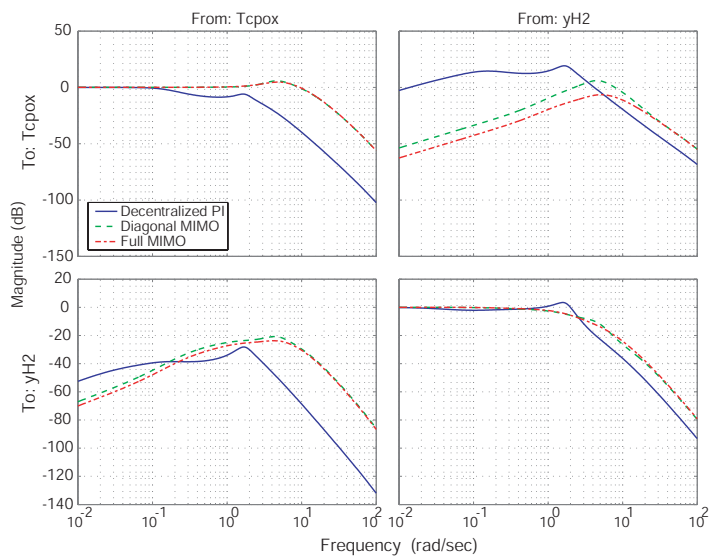

Figure 12: Frequency (magnitude in $\mathrm{dB}$ ) response from reference signal of closed loop system with MIMO controller

In summary, the MIMO controller achieves a superior performance in comparison with the decentralized PI controller due to two factors. First, the MIMO controller achieves a high bandwidth on the air loop without saturating the actuator by achieving high bandwidth without high gain. This is only feasible with high-order controllers. In hindsight of the success of the $C_{11}$ term of the MIMO controller, one can design a PID or a PI + lead-lag controller that reproduces similar gain and phase to be used in the decentralized controller.

Second, the MIMO controller achieves better coordination between the two actuators by utilizing a cross-coupling term. The cross-coupling term acts in a "feedforward" sense and changes the blower command based on how the fuel valve behaves. This partially cancels the interaction between the fuel valve to the air loop. This partial cancellation, luckily, involves plant elements that do not change significantly for different power levels. Thus, without having explicitly designed for robustness, the MIMO controller maintains its performance at all power levels. 


\section{Concluding Remarks}

The control problem of hydrogen generation using catalytic partial oxidation and prevention of fuel cell stack starvation is studied. The two-input two-output control problem has the air blower and the fuel valve as inputs and the CPOX temperature and the anode hydrogen mole fraction (anode starvation) as performance variables.

We show that tuning two PI controllers for the air and the fuel loops is difficult. Moreover, the closed loop performance is adversely affected by the intrinsic interaction between the two loops. One way to prevent the performance degradation is to have bandwidth separation between the two control loops. This introduces a compromise of the air-temperature closed loop response in favor to the fuel-hydrogen loop.

On the other hand, a model-based high-order controller designed using linear multivariable methodologies, LQR-LQG in our case, can achieve very good response for a wide range of operating conditions. Our analysis shows that the multivariable controller can be simplified to a lower triangular controller where the blower command depends on both errors in $T_{\text {cpox }}$ and $y_{H_{2}}$ (or, equivalently, fuel valve). If the multivariable controller is further simplified to a diagonal controller (no cross-coupling between control inputs and errors in the performance variables), the closed loop performance degrades with respect to the full multivariable controller but it still outperforms the two PI-based closed loop performance.

\section{Acknowledgements}

The authors would like to thank Thordur Runolfsson, Jonas Eborn, Christoph Haugstetter and Scott Bortoff at the United Technology Research Center for their help and valuable comments.

\section{References}

[1] A.L. Dicks, "Hydrogen generation from natural gas for the fuel cell systems of tomorrow," Journal of Power Sources, vol. 61, pp. 113-124, 1996.

[2] S. Ahmed and M. Krumpelt, "Hydrogen from hydrocarbon fuels for fuel cells," International Journal of Hydrogen Energy, vol. 26, pp. 291-301, 2001.

[3] L.F. Brown, "A comparative study of fuels for on-board hydrogen production for fuel-cell-powered automobiles," International Journal of Hydrogen Energy, vol. 26, pp. 381-397, 2001.

[4] V. Recupero, L. Pino, R.D. Leonardo, M. Lagana, and G. Maggio, "Hydrogen generator, via catalytic partial oxidation of methane for fuel cells," Journal of Power Sources, vol. 71, pp. 208-214, 1998

[5] J. Zhu, D. Zhang, and K.D. King, "Reforming of $\mathrm{CH}_{4}$ by partial oxidation: thermodynamic and kinetic analyses," Fuel, vol. 80, pp. 899-905, 2001.

[6] K. Ledjeff-Hey, J. Roses, and R. Wolters, " $\mathrm{CO}_{2}$-scrubbing and methanation as purification system for PEFC," Journal of Power Sources, vol. 86, pp. 556-561, 2000.

[7] D.Z. Megede, "Fuel processors for fuel cell vehicles," Journal of Power Sources, vol. 106, pp. 35-41, 2002.

[8] L. Pino, V. Recupero, S. Beninati, A.K. Shukla, M.S. Hegde, and P.Bera, "Catalytic partial-oxidation of methane on a ceriasupported platinum catalyst for application in fuel cell electric vehicles," Applied Catalysis A: General, vol. 225, pp. 63-75, 2002.

[9] T.E. Springer, R. Rockward, T.A. Zawodzinski, and S. Gottesfeld, "Model for polymer electrolyte fuel cell operation on reformate feed," Journal of The Electrochemical Society, vol. 148, pp. A11-A23, 2001.

[10] C.E. Thomas, B.D. James, F.D. Lomax Jr, and I.F. Kuhn Jr, "Fuel options for the fuel cell vehicle: hydrogen, methanol or gasoline?," International Journal of Hydrogen Energy, vol. 25, pp. $551-567,2000$.

[11] T.H. Gardner, D.A. Berry, K.D. Lyons, S.K. Beer, and A.D. Freed, "Fuel processor integrated $\mathrm{H}_{2} \mathrm{~S}$ catalytic partial oxidation technology for sulfur removal in fuel cell power plants," Fuel, vol. 81, pp. 2157-2166, 2002.

[12] A.L. Larentis, N.S. de Resende, V.M.M. Salim, and J.C. Pinto, "Modeling and optimization of the combined carbon dioxide reforming and partial oxidation of natural gas," Applied Catalysis, vol. 215, pp. 211-224, 2001.

[13] E.D. Doss, R. Kumar, R.K. Ahluwalia, and M. Krumpelt, "Fuel processors for automotive fuel cell systems: a parametric analysis," Journal of Power Sources, vol. 102, pp. 1-15, 2001.

[14] Jay Tawee Pukrushpan, Modeling and Control of Fuel Cell Systems and Fuel Processors, Ph.D. thesis, University of Michigan, 2003.

[15] C.R.H. de Smet, M.H.J.M. de Croon, R.J. Berger, G.B. Marin, and J.C. Schouten, "Design of adiabatic fixed-bed reactors for the partial oxidation of methane to synthesis gas. Application to production of methanol and hydrogen-for-fuel-cells," Chemical Engineering Science, vol. 56, pp. 4849-4861, 2001.

[16] R-H Song, C-S Kim, and D.R. Shin, "Effects of flow rate and starvation of reactant gases on the performance of phosphoric acid fuel cells," Journal of Power Sources, vol. 86, pp. 289-293, 2000.

[17] Sigurd Skogestad and Ian Postlethwaite, Multivariable Feedback Control: Analysis and Design, Wiley, 1996.

[18] Manfred Morari, Evanghelos Zafiriou, and Morari Zafiriou, Robust Process Control, Prentice Hall, 1997. 